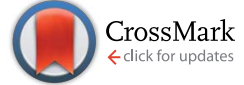

Cite this: RSC Adv., 2015, 5, 3814

Received 21st November 2014 Accepted 4th December 2014

DOI: 10.1039/c4ra14998j

www.rsc.org/advances

\section{Photophysics of lumichrome in anionic and cationic micellar media $\uparrow$}

\author{
Banibrata Maity, Aninda Chatterjee and Debabrata Seth*
}

The photophysics of lumichrome was studied in the presence of different cationic and anionic micellar environments. The change in ground and excited state spectral characteristics of lumichrome has been reported. Lumichrome showed excitation and emission wavelength dependent emission behaviour in micellar media. It is due to the presence of different species of lumichrome. The binding constants and free energy changes are dependent on the hydrophobic alkyl tail group of the surfactant. The time resolved area normalised emission spectra (TRANES) reveal the presence of an isoemissive point. It is due to two different emissive species present in the micellar media.

\section{Introduction}

Presently, photophysical studies on dyes in the presence of surfactants have drawn much importance due to their several applications in pharmaceutical science, luminescence, biochemistry, analytical chemistry, and applied science. ${ }^{1-6}$ The self-assembly of amphiphilic surfactant molecules in aqueous solution, (above a certain concentration of surfactant, known as critical micellar concentration [CMC]) forms thermodynamically stable 'molecular-clustered' called micelles. The surface active amphiphiles or surfactants are either ionic (cationic, anionic and zwetterionic) or non-ionic. The microenvironment of the fluorophore inside the micellar system is significantly different compared to the bulk solution..$^{7-9}$ The micropolarity of the water molecule gradually drops from the boundary surface to the core region inside the micelles. The most astonishing feature of organised assembly is that it has inclination to bind and stabilise the probe molecules that are insoluble or sparingly soluble in bulk solvents. The slow dynamics of water in micelles compared to the bulk solution is due to the specific interactions and confined geometry. ${ }^{10}$ The formation of micelles is governed by the decrease of free energy of the system due to divergence of hydrophobic tail segments from the aqueous periphery and reorganising of hydrogen bond network in water. This type of additional energy gain is responsible for van der Waals bond formation between hydrophobic pockets in the core of the micelles. ${ }^{11,12}$

Flavins, generally consists of tricyclic heteronuclear organic moiety, are found in many biological systems and undergo important redox reactions, electron transfer and

Department of Chemistry, Indian Institute of Technology Patna, Patna 800013, Bihar, India. E-mail: debabrata@iitp.ac.in; Fax: +91-612-2277383

$\dagger$ Electronic supplementary information (ESI) available. See DOI: 10.1039/c4ra14998j signal transduction. ${ }^{\mathbf{1 3 , 1 4}}$ Lumichrome (LCM) is one of the most important archetype model chromophore in flavin members related to lumazine and isoalloxazines. LCM act as triplet photosensitizers, originate singlet oxygen $\left({ }^{1} \mathrm{O}_{2}\right)$ and auspicate oxidation of many living organs like enzymes, proteins, nucleic acids and hormones. ${ }^{15,16}$ This molecule suppresses the flavin reductase in living Escherichia coli cells and prevents food poisoning. ${ }^{16} \mathrm{LCM}$ consists of both hydrogen bond donor and acceptor centres ( $-\mathrm{O}$ atoms, $-\mathrm{N}$ atoms, $-\mathrm{N}-\mathrm{H}$ groups) and nitrogen-unsubstituent alloxazine scaffold and undergoes tautomerisation process via $\mathrm{N}(1)$ to $\mathrm{N}(10)$ atoms. DMAL species (7,8-dimethyl alloxazine) is the ground state analogue. The excited state proton-transfer (ESPT) occurs via tautomerisation process. It forms isoalloxazine scaffold DMIS species (7,8-dimethyl isoalloxazine) in the presence of hydrogen bond donating molecules such as pyridine derivatives, acetic acid. ${ }^{17,18}$ DMAL is the most ascendant species in ground state and DMIS is formed in the excited state. LCM converted from alloxazine to isoalloxazine species in the presence of mixed solvents like acetic acid-ethanol and dioxane-pyridine mixtures. ${ }^{19-21}$ Biczók and co-worker reported that LCM undergoes tautomer formation in ground state in the presence of cucurbit[7]uril and strong hydrogen bond acceptor anions (such as fluoride, acetate anion). ${ }^{22,23}$ LCM is the major product of photodecomposition of riboflavin (vitamin $\mathrm{B}_{2}$ ) under neutral or acidic pH. Using quantum mechanical calculation, Sikorski et al. ${ }^{\mathbf{2 4}}$ found an excited state double proton transfer (ESDPT) occurs in the LCM dimer in the solid state. A different type acid-base equilibrium of LCM at different $\mathrm{pH}$ values suggested the existence of different species LCM such as neutral species (DMAL and DMIS), two monoanions, and one dianion species. ${ }^{25,26}$ The photophysics of LCM has been subjected to intense research in homogeneous media and in numerous bio-mimicking confined media over the years. ${ }^{27-34}$ 
We reported the photophysics of LCM in the presence of aqueous and non-aqueous reverse micelles. ${ }^{34}$ Here, in this perspective we are interested to know the effect of confinement on the emission behaviour of LCM in the presence of various cationic and anionic micellar media. The objective of our study is to show the effect of different-charged surfactants and nature of the polar head group of the micelles on the photophysics of LCM. The dependence of the hydrophobic alkyl chain lengths of the surfactants on the photophysics of LCM was discussed. The different species of LCM present in different cationic and anionic micelles was also discussed. The excitation and emission wavelength dependent fluorescence dynamics of LCM in micelles was discussed.

\section{Experimental section}

\subsection{Materials}

LCM (Scheme 1) was purchased from Sigma-Aldrich and used as received. The surfactants (Scheme 1) sodium dodecyl sulphate (SDS), sodium octyl sulphate (SOS), dodecyl trimethyl ammonium bromide (DTAB), myristyl trimethyl ammonium bromide (MTAB) and cetyl trimethyl ammonium bromide (CTAB) were purchased from Sigma-Aldrich and were used as received. All chemicals were of the highest available purity and used as received. Millipore water was used for preparation of all solutions. The concentration of LCM in the solution was maintained $2 \times 10^{-5} \mathrm{M}$. The final concentrations of SDS, DTAB, MTAB and CTAB surfactants were maintained $\sim 20$ times of their critical micellar concentration (CMC) value. The concentration of SOS was maintained $\sim 3$ times of CMC to ensure the formation of micelles.
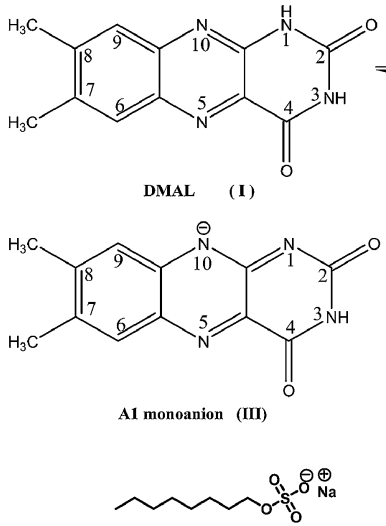

$\operatorname{SOS}(\mathrm{n}=8)$

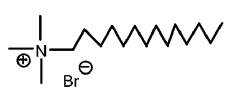

$\operatorname{DTAB}(\mathrm{n}=12)$

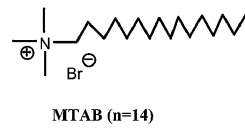

( $\mathrm{n}=$ alkyl chain length)

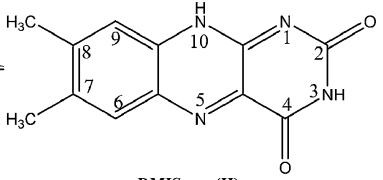

DMUS (II)
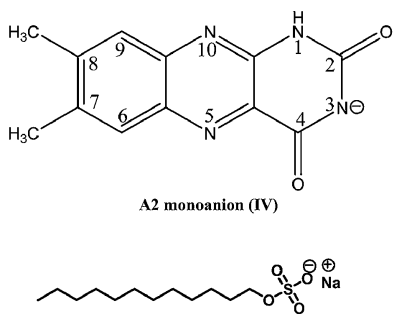

$\operatorname{SDS}(\mathrm{n}=12)$

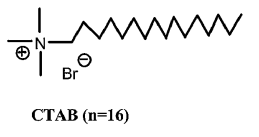

Scheme 1 Schematic representation of LCM in neutral (DMAL and DMIS) and anionic (A1 and A2 monoanion) species and different anionic and cationic surfactants.

\subsection{Instrumentation and methods}

The steady-state absorption and fluorescence emission measurements were done using ultraviolet-visible (UV-Vis) spectrophotometer (Model: UV-2550, Shimadzu) and spectrofluorometer (Model: Fluoromax-4P, Horiba Jobin Yvon) respectively. For absorption and fluorescence measurements the path length of the used quartz cuvette was $1 \mathrm{~cm}$. All the steady state measurements were carried out at $298 \mathrm{~K}$ by using a refrigerated bath circulator (Model: RW0525G, Jeoitech). The fluorescence quantum yield of LCM in micellar media were measured using quinine sulphate solution in $0.1(\mathrm{~N}) \mathrm{H}_{2} \mathrm{SO}_{4}\left(\Phi_{\mathrm{R}}\right.$ $=0.546)$ as reference. ${ }^{35} \mathrm{We}$ have calculated the quantum yield $\left(\Phi_{\mathrm{S}}\right)$ value by using the following equation:

$$
\Phi_{\mathrm{S}}=\Phi_{\mathrm{R}}\left(\frac{A_{\mathrm{S}}}{A_{\mathrm{R}}} \times \frac{\mathrm{Abs}_{\mathrm{R}}}{\mathrm{Abs}_{\mathrm{S}}} \times \frac{n_{\mathrm{S}}^{2}}{n_{\mathrm{R}}{ }^{2}}\right)
$$

where, ' $\Phi$ ' represents the quantum yield, ' $A$ ' is the integrated area under the fluorescence curve, 'Abs' is absorbance of the respective solution at the excitation wavelengths and ' $n$ ' stands for refractive index of the medium. The subscript ' $S$ ' and ' $R$ ' stands for experimental sample and reference respectively.

The fluorescence lifetime decays were collected by using picosecond time-correlated single photon counting (TCSPC) technique. We have used a time-resolved fluorescence spectrophotometer from Edinburgh Instruments (model: LifeSpec-II, UK). The samples were excited at $375 \mathrm{~nm}, 405 \mathrm{~nm}$ and 445 $\mathrm{nm}$ by using picosecond diode laser. The signals were collected at magic angle $\left(54.7^{\circ}\right)$ using Hamamatsu MCP PMT (3809U) as detector. The instrument response function of our setup is 80 ps. The analyses of the decays were done using F-900 decay analysis software. All experiments were carried out three times to check the reproducibility of the data. In the case of time resolved measurement, the temperature was maintained at 298 K by using a Peltier-controlled cuvette holder (Model: TLC-50, Quantum Northwest).

\section{Results and discussion}

\subsection{Steady state absorption studies}

The UV-Vis absorption spectral studies of LCM were carried out in the presence of cationic and anionic micelles. LCM exhibits two characteristic absorption peaks in the aqueous solution, it was also reported in the literature. ${ }^{27-29,34}$ The first absorption maximum appears at $353 \mathrm{~nm}$ along with a shoulder at $385 \mathrm{~nm}$. The two absorption maxima of LCM are due to two independent $\pi-\pi *$ transitions of neutral DMAL species of LCM. ${ }^{36}$ In addition, we have also observed a tail in the absorption spectra (Fig. 1a) of LCM, extends upto $\sim 450 \mathrm{~nm}$, in the presence of pure water and micelles. Similar kind of behaviour was also reported by Douhal and co-workers. ${ }^{31}$ In the presence of micelles, we observed that the first absorption maximum undergoes blue shift (Table 1) compared to that in pure water, while the position of shoulder band remains almost fixed. Noticeably, the first absorption maximum was blue shifted by $11 \mathrm{~nm}$ in the presence of SOS micelles compared to pure water. Similarly, for SDS micelle, the first absorption maximum was observed at $349 \mathrm{~nm}$. Moreover, 

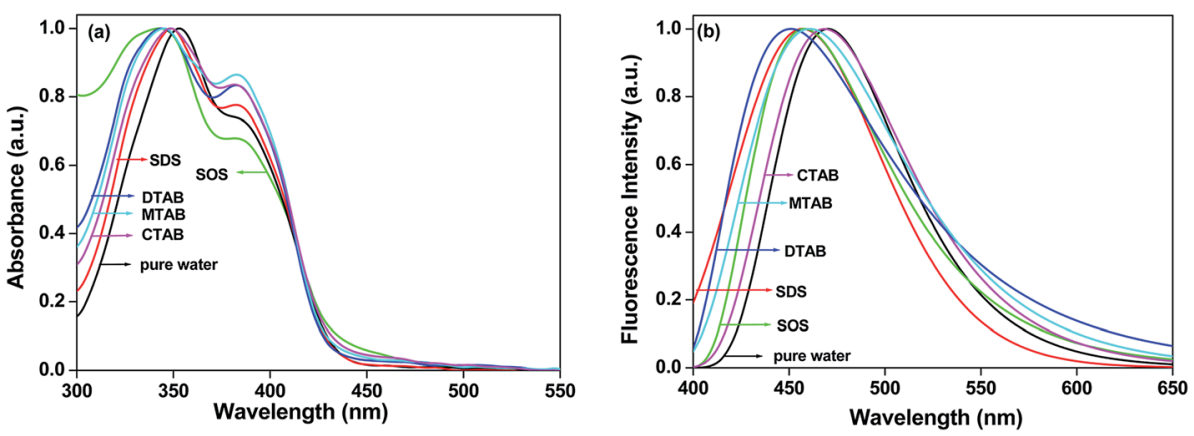

Fig. 1 (a) The change in absorption and (b) emission spectral position of LCM in different micellar media and in pure water.

the first absorption maximum was blue-shifted compared to pure water and appeared at $344 \mathrm{~nm}, 345 \mathrm{~nm}$ and $349 \mathrm{~nm}$ in DTAB, MTAB and CTAB micelles respectively, when concentration of the surfactants reached 20 times of their CMC values. This result indicates that the environment of fluorophore gets modified in the presence of micellar media compared to the aqueous solution. Upon successive addition of surfactants in the aqueous solution of LCM, the intensity of the first absorption band slightly decreases and a hypsochromic shift was observed. This result showed the interaction of LCM molecule with the respective micelles in the ground state. Fig. 1a displays modification of the absorption spectra of LCM in the presence of both cationic and anionic micelles compared to pure water. The weak absorption tail found at $\sim 450 \mathrm{~nm}$ in the presence of pure water and micelles is due to small population of the isoalloxazine species of LCM anion. ${ }^{31}$ This clearly reflects that the location of fluorophore gets modified with addition of surfactant in the aqueous solution of LCM.

\subsection{Steady state fluorescence emission studies}

In pure water, LCM shows emission maximum at $471 \mathrm{~nm}$, and the peak position remains unchanged upon varying the excitation wavelengths. We have studied the emission spectral characteristics of LCM in different micelles to know the influence of micellar environments on the emission spectra. With increase in the concentration of anionic surfactants the fluorescence intensity of LCM is quenched. But this effect is less prominent compared to the cationic surfactants. In both cases, we have found a hypsochromic shift on the emission spectra of LCM in different micellar environment as shown in the Table 1 and
Fig. 1b. These suggest that LCM sensed less polar environment inside the micellar environment compared to the aqueous media. In case of SOS micelle, the emission peak is blue-shifted by $13 \mathrm{~nm}$ compared to the aqueous solution. In SDS micelles, the emission maximum of LCM is shifted from $471 \mathrm{~nm}$ (in aqueous solution) to $457 \mathrm{~nm}$ ([SDS] $=20$ times of CMC). Therefore, in the presence of anionic micelles it is evident that the proximity of peak position is same. This reveals that the micropolarity around the probe inside the anionic micellar environment remains almost identical. The emission peak position of LCM in cationic micelles is blue shifted compared to pure water. The fluorescence intensity also gradually decreases as shown in Fig. 2. The emission peak positions of LCM were found at $450 \mathrm{~nm}, 461 \mathrm{~nm}$ and $468 \mathrm{~nm}$ in DTAB, MTAB and CTAB micellar environment, respectively. This states that polarity of the medium plays a crucial role. This is true for all micellar media studied by us and it can be ratiocinate in terms of binding of LCM molecule with less polar micellar media as compared to the aqueous medium. Moreover, we found that the quantum yield of LCM in pure water is 0.063 (ref. 34) and this value decreases in the presence of cationic surfactants as shown in Table 1. The change of quantum yield of LCM follows the order $\mathrm{DTAB}>\mathrm{MTAB}>\mathrm{CTAB}$. It is pointed out that with gradual increase of the alkyl chain length of the surfactant the quantum yield value gradually increases. In the case of anionic surfactants, the quantum yield value of LCM in SOS and SDS are 0.039 and 0.053 respectively. The decrease of the fluorescence quantum yield and the blue shift of emission spectra of LCM in anionic micelles compared to that in aqueous solution, suggest that the environment around the probe molecule in the micellar solutions get modified compared to that in pure water. The low

Table 1 Photophysical parameters and binding constants of LCM with different micelles

\begin{tabular}{|c|c|c|c|c|c|c|c|c|}
\hline Surfactants & $\lambda_{\max }^{\mathrm{abs}}(\mathrm{nm})$ & $\begin{array}{l}\lambda_{\max }^{\mathrm{emi}} \\
(\mathrm{nm})\end{array}$ & $\begin{array}{l}\lambda_{\text {exi }} \\
(n m)\end{array}$ & $\begin{array}{l}\mathrm{CMC}^{36,37} \\
\text { (in } \mathrm{mM} \text { ) }\end{array}$ & $\Phi_{\mathrm{f}}$ & $\left\langle\tau_{\mathrm{f}}\right\rangle^{a}(\mathrm{~ns})$ & $\chi^{2}$ & $\begin{array}{l}\text { Binding constant } \\
(K)\left(\text { in } \mathbf{M}^{-1}\right)\end{array}$ \\
\hline $\operatorname{SOS}(n=8$, anionic $)$ & 342,385 & 458 & \multirow[t]{4}{*}{375} & 134 & 0.039 & 1.40 & 1.03 & $2.18 \times 10^{3}( \pm 120)$ \\
\hline $\operatorname{DTAB}(n=12$, cationic $)$ & 344,384 & 450 & & 15.6 & 0.006 & 0.16 & 0.85 & $5.80 \times 10^{3}( \pm 300)$ \\
\hline $\operatorname{MTAB}(n=14$, cationic $)$ & 345,384 & 461 & & 4.2 & 0.011 & 0.28 & 0.91 & $13.79 \times 10^{3}( \pm 455)$ \\
\hline CTAB $(n=16$, cationic $)$ & 349,383 & 468 & & 0.92 & 0.022 & 0.87 & 0.96 & $26.00 \times 10^{3}( \pm 1035)$ \\
\hline
\end{tabular}

${ }^{a}\left\langle\tau_{\mathrm{f}}\right\rangle=a_{1} \tau_{1}+a_{2} \tau_{2}+a_{3} \tau_{3}$. 

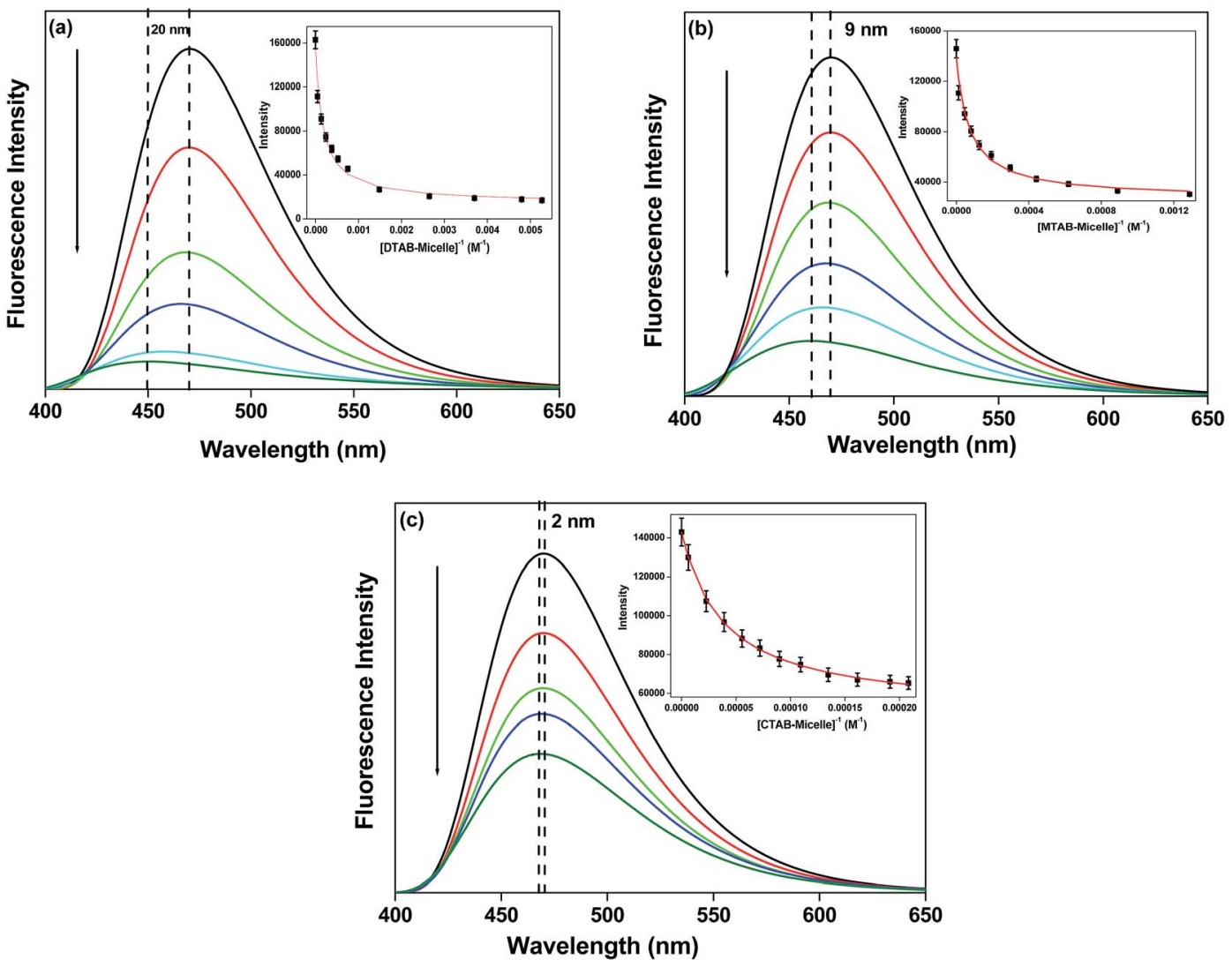

Fig. 2 The emission spectral profile of LCM $\left(\lambda_{\text {exi }}=375 \mathrm{~nm}\right.$ ) in cationic (a) DTAB, (b) MTAB and (c) CTAB micelles. Inset shows the variation of fluorescence intensity against respective [Micelle].

emission quantum yield of LCM in the presence of different micelles maybe due to low polarity of the micellar system compared to bulk solution. Similar behaviour of LCM in the presence of other confined media was reported in the literature. ${ }^{31,32,34}$ It is evident from Table 1 that on going from DTAB, MTAB and CTAB micelles, the extent of quenching of fluorescence emission decreases as evident from fluorescence quantum yield and average lifetime values. Similar type of observation is also found in case anionic micelles where, the extent of fluorescence quenching decreases from SOS to SDS micelles. These results indicate that with increasing hydrophobic alkyl chain length of the surfactants, the efficiency of fluorescence quenching phenomenon decreases.

\subsection{Steady state fluorescence excitation spectra}

The normalised fluorescence excitation spectra (monitored at $450 \mathrm{~nm}$ and $570 \mathrm{~nm}$ ) of LCM in pure water and in anionic and cationic micelles are shown in Fig. 3 and S1. $\dagger$ It shows the presence of different species of LCM. The shape of the normalised fluorescence excitation spectra, monitored at $450 \mathrm{~nm}$, is similar to absorption spectra of LCM. This observation in pure water and in the micelles indicates that the alloxazine (DMAL) species of LCM is the predominant species in the emission maximum region. Significant increase of the shoulder intensity around $430-460 \mathrm{~nm}$ region was observed, when the fluorescence excitation spectra was recorded at longer wavelength $\left(\lambda_{\text {emi }}=570 \mathrm{~nm}\right)$. When excited state proton transfer (ESPT) occurs, then the excitation spectrum of LCM should be invariant of the emission wavelength, because it is only alloxazine species that absorbs the light. However, when there is already tautomerism in the ground state, then there are two emitting species present. Then the excitation spectra must exhibit changes as we have observed in our experiments. The weak tail around the long wavelength region in the absorption spectra is due to the different species of LCM exists in pure water and micelles. Therefore, from the absorption, emission and excitation spectra, of LCM in pure water and micelles indicate two component behaviour of LCM (alloxazine and isoalloxazine). In presence of pure water, LCM molecule is unable to tautomerise and therefore the formation of tautomeric neutral isoalloxazine species of LCM in the ground state and the excited state can be excluded based on fluorescence excitation spectra as well as the literature reports. ${ }^{22,27,31}$ Therefore, in presence of pure water, two different species of LCM are neutral alloxazine species (DMAL) and deprotonated monoanionic A1 species. ${ }^{31}$ Similar kind of the observation on LCM in pure water was also reported in the literature. ${ }^{31}$ In presence of the studied micellar system, the tautomeric species of LCM may also present in the ground state and therefore we have found different excitation spectra, which are independent of emission wavelength. It is well known that interfacial $\mathrm{pH}$ of micelle-water 

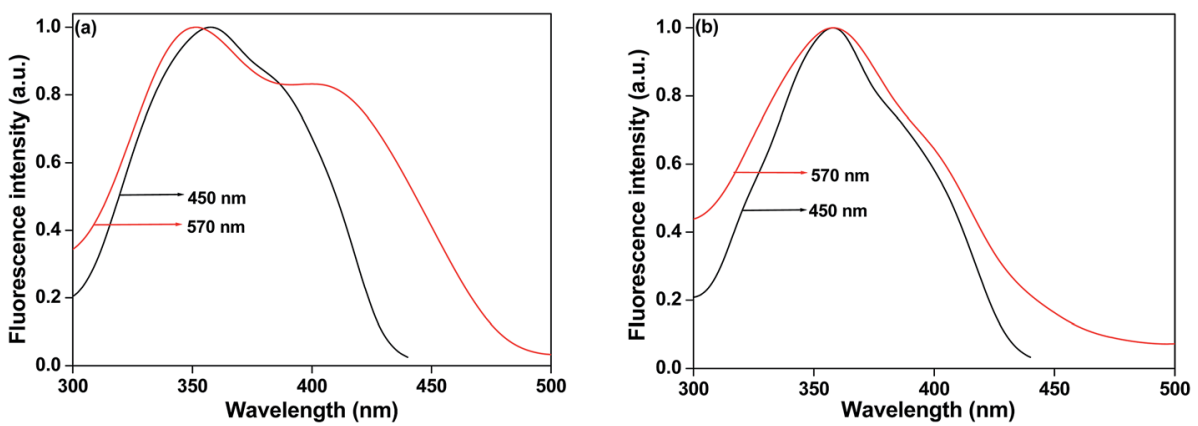

Fig. 3 The normalised fluorescence excitation spectra of LCM in (a) SOS (anionic) and (b) CTAB (cationic) micelles. The observation wavelengths were at $450 \mathrm{~nm}$ (black) and $570 \mathrm{~nm}$ (red).

is different compared to bulk $\mathrm{pH}$. The $\mathrm{pH}$ of SDS and СTAB micelle-water interface are $<7$ and 10.8 respectively. ${ }^{37,38}$ Therefore, in our studied micellar systems we may assume that $\mathrm{pH}$ of micelle-water interface lies in between $7>\mathrm{pH}<11$. Sikorski et $a l .{ }^{27}$ reported earlier that $6<\mathrm{pH} \leq 10$, the neutral and monoanionic species of LCM molecule coexists. Therefore, in our systems, we may also assume that both the neutral (tautomeric form) and the monoanionic species of LCM coexist. ${ }^{25,27}$

\subsection{Wavelength sensitive fluorescence emission behaviour of LCM}

We have observed excitation wavelength dependent fluorescence emission behaviour of LCM in aqueous solution. Douhal and co-workers ${ }^{31}$ also observed excitation wavelength dependent behaviour of LCM in water at $\mathrm{pH}$ 6.7, suggesting two emissive species is formed in solution. The excitation wavelength dependent fluorescence emission behaviour of LCM entrapped in different micellar environments has been observed. The emission peak position of LCM at some selected excitation wavelengths are tabulated in Table 2. In SOS micelle we have observed $54 \mathrm{~nm}$ red shifting of the emission peak position of LCM by changing the excitation wavelength from $375 \mathrm{~nm}$ to $445 \mathrm{~nm}$ (Fig. 4a). This phenomenon maybe due to the existence of different emitting species of LCM present in the aforesaid medium. Feitelson and co-workers ${ }^{25}$ proposed that in aqueous solution, due to interaction of LCM molecule with water, it form $\mathrm{H}$-bond and produce deprotonated anionic species of LCM. Therefore, it can be assumed that the stable DMAL is the predominant species, with minor population of deprotonated monoanionic species. The origins of such excitation wavelength dependent fluorescence emission maybe due to different species of LCM present in micelles. In SDS micelle, the excitation wavelength dependent emission behaviour is also observed. The shift in emission peak position is $23 \mathrm{~nm}$ (Fig. S2a $\dagger$ ). In the case of DTAB, MTAB and CTAB micelles, the shift of emission peak positions are $63 \mathrm{~nm}, 52 \mathrm{~nm}$ and $46 \mathrm{~nm}$ respectively, with changing the excitation wavelength from 375 $\mathrm{nm}$ to $445 \mathrm{~nm}$ (Fig. 4 and S2 $\dagger$ ). Chen et al. ${ }^{39}$ found that the emission peak position of quinine is strongly dependent on the excitation wavelength and gave an explanation that distinct emission peak arises due to two different species of the molecule. Since, different species of LCM exists. So, we may interpret that the reason of excitation wavelength dependent fluorescence emission behaviour is maybe due to different species of the LCM molecule in the micellar media.

\subsection{Probe-micelles binding study}

With increase of hydrophobic alkyl chain length of the surfactant molecule, the hydrophobicity of the micellar medium increased. For this reason tendency of aggregation of surfactant molecules are increased and it reduced the CMC value. To elucidate, how potently a probe molecule binds with micelles, we have determined the binding constant value of LCM with micelles. The binding constant $(K)$ values have been determined from the variation of fluorescence intensity against the concentration of different micelles using the $1: 1$ nonlinear least-squares regression analysis method: ${ }^{40}$

$$
F=\frac{F_{\text {water }}+F_{\text {micelle }} K_{1}[\text { Micelle }]}{1+K_{1}[\text { Micelle }]}
$$

where, $F_{\text {water }}$ and $F_{\text {micelle }}$ are the fluorescence intensities of LCM in water and in different micelles, when complete binding of dye with the micelle has occurred. $K_{1}$ is the binding constant.

The micellar concentration is determined by:

$$
[\text { Micelle }]=\frac{(S-\mathrm{CMC})}{N}
$$

where, ' $S$ ' represents the concentration of respective surfactant under experimental condition, $\mathrm{CMC}$ is the critical micellar concentration and ' $N$ ' stands for the aggregation number of the micellar system. The values of CMC and ' $N$ ' have taken from literature. ${ }^{41-43}$ The binding constant value $(K)$ have been determined from the plot of variation of fluorescence intensity against the concentration of different micelles (shown in inset of Fig. 2 and S3†). From Table 1, we have found that in SOS micelle, the binding constant value $(K)$ is $2.18 \times 10^{3} \mathrm{M}^{-1}$ whereas, in CTAB micelles this value enhances $\sim 12$ times. It has been noticed that the binding constant value gradually increases with increase of hydrophobic chain length of the surfactants, for both anionic and cationic surfactants. The binding ability is strong in the case of cationic micelles compared to the anionic micelles as evidenced from the binding constant value. It has been observed that binding constant is maximum in the case of CTAB micelle and 
Table 2 The components of time resolved emission decays of LCM in micelles at different excitation and emission wavelengths

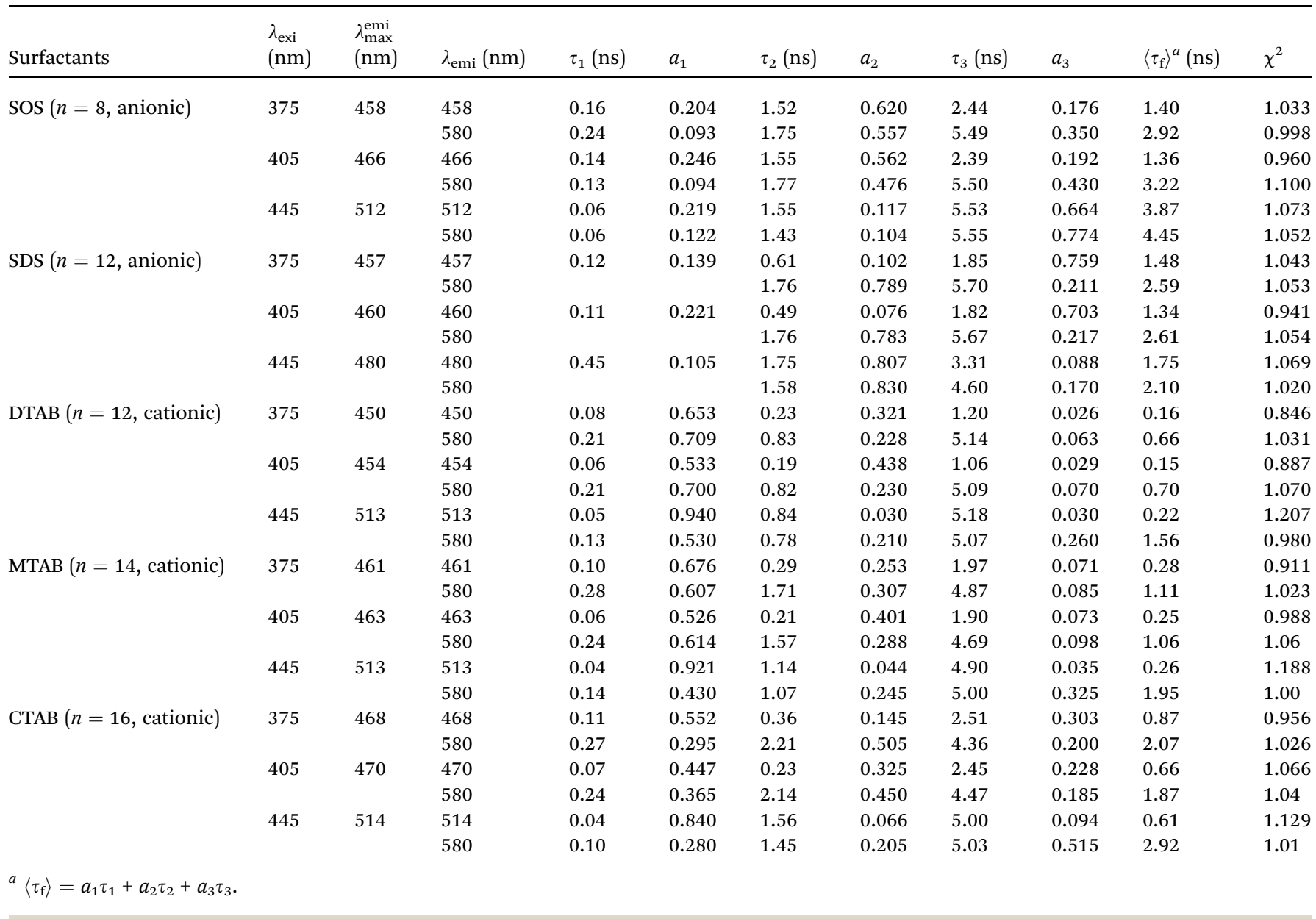

minimum in the case SOS micelle. This maybe due to degree of penetration of the fluorophore is more inside the cationic micelles compared to anionic micelles. Moreover, the water content is rich in anionic micellar periphery compared to cationic micelles. ${ }^{4}$

In the excited state of LCM, proton transfer process is facile. $^{17,29}$ From quantum chemical calculation ${ }^{36}$ it was disclosed that the molecule exist as an anionic species. In case of cationic micelles, the strong electrostatic force between the positive polar head groups and LCM is operated. The hydrogen bonding interaction of water in the Stern-layer with negatively charged LCM causes strong binding. With gradual increase of hydrophobic chain length of the surfactants, the hydration layer becomes drier and strengthens the electrostatic interaction.

\subsection{Time resolved fluorescence emission study}

The excited state lifetime of the probe molecule is a sensitive indicator for analysing the local environment around the
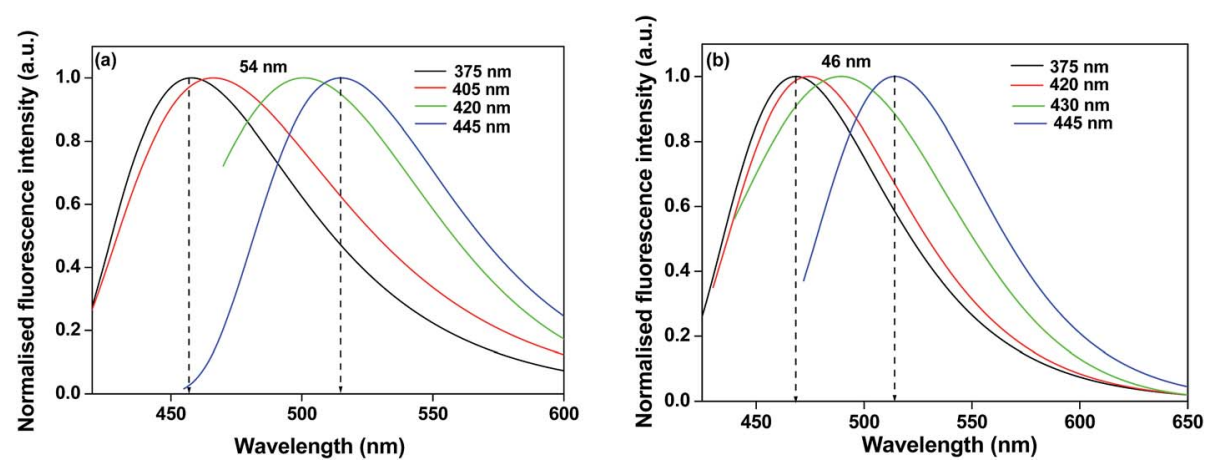

Fig. 4 The excitation wavelength dependent emission spectral profile of LCM in (a) SOS and (b) CTAB micelles. 
fluorophore and it gives an idea about the excited state behaviour of LCM. To elucidate the microenvironment around the fluorophore, we have studied the time resolved fluorescence emission behaviour of LCM in micellar media. The fluorescence emission decays of LCM in different micellar media are fitted by three exponential functions. The fluorescence emission decays of LCM in the presence of micelles are found to be dependent on both the excitation and emission wavelength. With successive addition of surfactants beyond their respective CMC values, the relative contribution of both the first component and second component gradually increases whereas, the long component drastically decreases. This result gets support that the photophysics of LCM in the presence of micellar media is modified compared to aqueous media. The emission decays of LCM in different micelles and in pure water are shown in Fig. 5 .

Fig. 6a shows a comparison of emission decays of LCM in pure water and in the presence of SOS micelle. LCM molecule bound to anionic SOS micelle shows three lifetime components, $\tau_{1}(0.16 \mathrm{~ns}), \tau_{2}(1.52 \mathrm{~ns})$ and $\tau_{3}(2.44 \mathrm{~ns})$, with average lifetime value of $1.40 \mathrm{~ns}$ (when $\lambda_{\text {exi }}=375 \mathrm{~nm}$ ). The $\tau_{1}$ component is due to the interaction of LCM anion with micellar surface and its relative population is $\sim 20 \%$. Similar type of component was reported by Douhal and co-workers. ${ }^{31}$ and proposed that this component is due to different interaction of LCM with HSA protein. The second component $\left(\tau_{2}\right)$ is due to the excited state of the complexed alloxazine species (LCM-SOS) with its relative

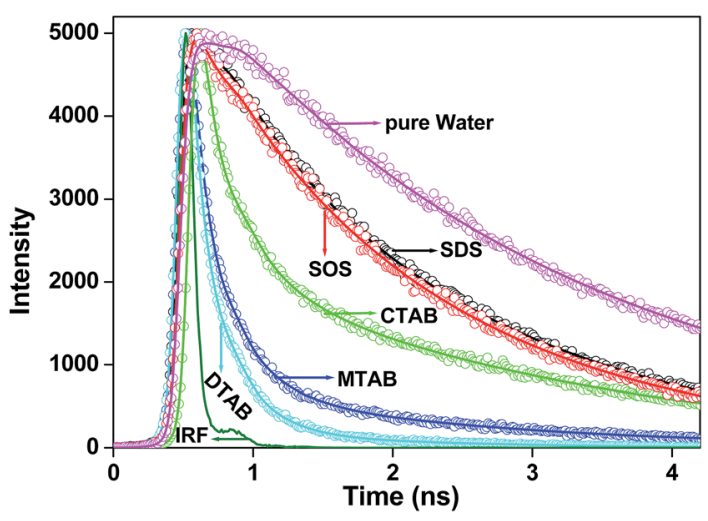

Fig. 5 The time resolved fluorescence emission decays of LCM in different micellar media and in pure water. population $62 \%$. Similar type of component also reported by Biczók and co-workers ${ }^{22}$ in the presence of cucurbit[7]uril. The third component $\left(\tau_{3}\right)$ is slightly changed from $2.7 \mathrm{~ns}$ to $2.44 \mathrm{~ns}$ and its relative population is $18 \%$. The second component $\left(\tau_{2}\right)$ and the third component $\left(\tau_{3}\right)$ looks like quite identical because of the insufficient lifetime difference of LCM-SOS complex and free LCM molecule. Biczók and co-workers ${ }^{22}$ reported that emission decay from the two coexisting emissions cannot be resolved and as a result we have found the lifetime component is quite different as compared to the LCM-SOS complex and free LCM molecule (lifetime $2.7 \mathrm{~ns}$ ). Monitoring the fluorescence decay from emission peak maxima to red end $(580 \mathrm{~nm})$, the magnitude of $\tau_{3}$ component changes from $2.44 \mathrm{~ns}$ to $5.49 \mathrm{~ns}$ and relative population gradually enhances. Biczók and coworkers $^{22}$ reported similar type of $5.1 \mathrm{~ns}$ component of LCM in presence of $\mathrm{CB} 7$ and they assigned this component is due to the tautomer-CB7 complex. Therefore, in our case we may also assume that $5.49 \mathrm{~ns}$ component is due to the tautomer-SOS complex. Briefly, we can attribute that in presence of SOS micelles, LCM molecule induces structural change from alloxazine to isoalloxazine species. The emission wavelength dependent lifetime decays of LCM $\left(\lambda_{\text {exi }}=375 \mathrm{~nm}\right)$ in the presence of SOS micelles are shown in Fig. 7a. When, $\lambda_{\text {exi }}=405 \mathrm{~nm}$, we have found almost similar type of components and relative amplitude, suggesting that no significant change of the species of LCM by varying excitation wavelength from $375 \mathrm{~nm}$ to 405 $\mathrm{nm}$. When $\lambda_{\text {exi }}=445 \mathrm{~nm}$, first two components $\left(\tau_{1}\right.$ and $\left.\tau_{2}\right)$ remain almost same but, the slow component $\left(\tau_{3}\right)$ is remarkably changed and found a $5.53 \mathrm{~ns}$ component. This is also assigned for the tautomer-SOS complex of LCM present in the excited state. For all excitation wavelengths, we have found $\sim 5.5 \mathrm{~ns}$ component, when emission was collected at the red end, suggesting the existence of tautomer-SOS complex.

In the case of other micelles we have found similar type behaviour of LCM molecule. The emission decays of LCM gradually quenched with the gradual addition of respective surfactants as shown in Fig. 6 and S4. $\dagger$ The lifetime components and their relative amplitudes of LCM in presence of different micelles are tabulated in Table 2. In all the cases we have found tautomer-micelles species of LCM exists in micellar media. In presence of SDS, DTAB, MTAB and CTAB micelles the average lifetime values of LCM are $1.48 \mathrm{~ns}, 0.16 \mathrm{~ns}, 0.28 \mathrm{~ns}$ and $0.87 \mathrm{~ns}$ respectively (when $\lambda_{\text {exi }}=375 \mathrm{~nm}$ ). In SDS micelles, the
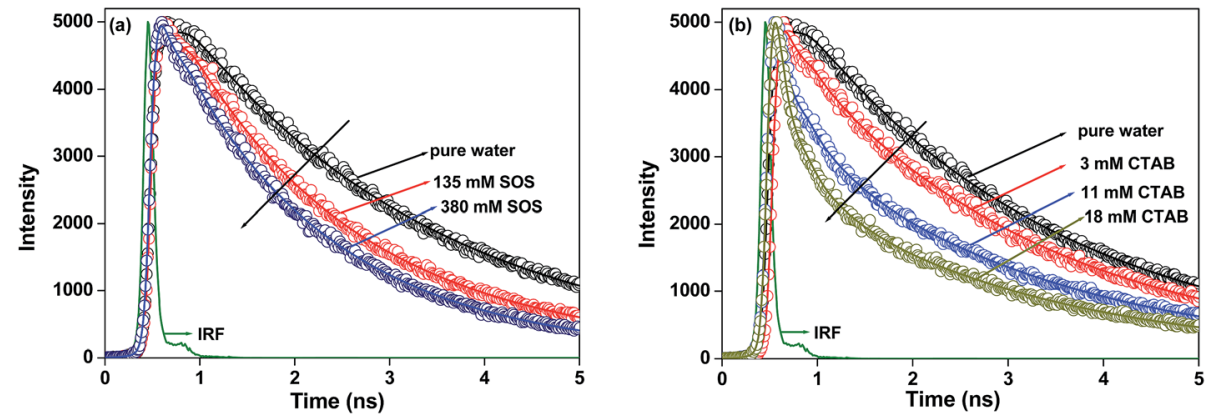

Fig. 6 The time resolved fluorescence emission decays of LCM with gradual addition of (a) SOS, (b) CTAB. 

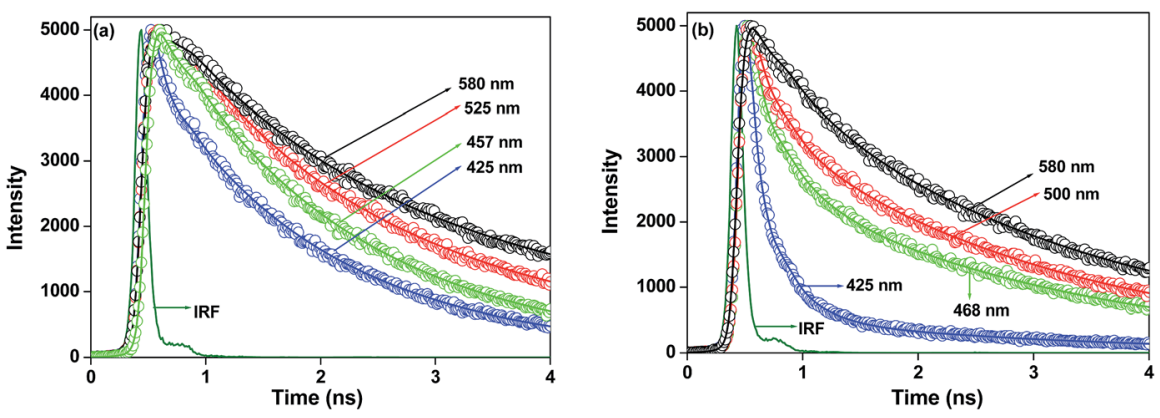

Fig. 7 The emission wavelength dependent lifetime decays of LCM in (a) SOS and (b) CTAB micelles.

three components are $0.12 \mathrm{~ns}\left(\tau_{1}\right), 0.61 \mathrm{~ns}\left(\tau_{2}\right)$ and $1.85 \mathrm{~ns}\left(\tau_{3}\right)$ and their relative amplitudes are $14 \%, 10 \%$ and $76 \%$ respectively. The components $\tau_{1}$ and $\tau_{2}$ can be presumed due to the different type of interaction of anionic LCM with the micellar media. Douhal and co-workers ${ }^{31}$ also observed $0.14 \mathrm{~ns}$ and 0.83 ns components of LCM in the presence of HSA protein and presumes the components are due to different interactions of the anions with the protein. The long component $\left(\tau_{3}\right)$ is due to the excited state of the complexed alloxazine species ${ }^{22}$ which is deviated from the lifetime decay of free LCM molecule (lifetime of DMAL species of LCM is $2.7 \mathrm{~ns}$ ). In DTAB micelles, the three components of LCM are $0.08 \mathrm{~ns}\left(\tau_{1}\right), 0.23 \mathrm{~ns}\left(\tau_{2}\right)$ and $1.2 \mathrm{~ns}\left(\tau_{3}\right)$ and their relative amplitudes are $65 \%, 32 \%$ and $3 \%$ respectively. The long component $\left(\tau_{3}\right)$ is due to the monoanionic A2 species of LCM and its relative population is very small. Feitelson and co-workers ${ }^{25}$ and Douhal and co-workers ${ }^{31}$ found similar type of long component from emission decay of LCM at $\mathrm{pH} \sim 10.3$. In presence of MTAB micelles $\left(\lambda_{\text {exi }}=375\right)$, the lifetime components are $0.1 \mathrm{~ns}\left(\tau_{1}\right), 0.29 \mathrm{~ns}\left(\tau_{2}\right)$ and $1.97 \mathrm{~ns}\left(\tau_{3}\right)$ with relative population $\sim 68 \%, 25 \%$ and $7 \%$ respectively. The third component $\left(\tau_{3}\right)$ maybe due to excited state of the complexed alloxazine species. ${ }^{22}$ In CTAB micelles, the lifetime components of LCM are $0.11 \mathrm{~ns}\left(\tau_{1}\right), 0.36 \mathrm{~ns}\left(\tau_{2}\right)$ and $2.51 \mathrm{~ns}\left(\tau_{3}\right)$ with relative populations are $55 \%, 15 \%$ and $30 \%$ respectively. The long component $\left(\tau_{3}\right)$ is also deviates from the emission decay of DMAL species of LCM (lifetime $2.7 \mathrm{~ns}$ ) and it maybe assigned as excited state of the complexed alloxazine species. ${ }^{22}$ Similar type of structural change from alloxazine (normal DMAL species of LCM) to the isoalloxazine species (either in ionic or the neutral tautomeric species) of LCM exists in the presence of different micelles was observed from the excitation and emission wavelength dependent behaviour. Pill-Soon-Song et al. have reported that in presence of pyridine-dioxane and acetic acid-ethanol mixtures, the alloxazine species of LCM undergoes excited state proton transfer via $\mathrm{N}-1$ to $\mathrm{N}-10$ atom to form isoalloxazine species. ${ }^{19,20}$ Biczók et al. also reported that in presence of cucurbit[7]uril, the proton transfer of LCM takes place due to phototautomerisation process. ${ }^{22}$ In our case, we may assume that in the excited state of LCM, proton transfer takes place in micelles as we have obtained one isoemissive point. This clearly indicates the presence of tautomeric species of LCM in the micelles. We have obtained two distinct emission peaks (470 $\mathrm{nm}$ and $\sim 515 \mathrm{~nm}$ ) with variation of the excitation wavelength.
The two distinct emission peaks are due to alloxazine $(470 \mathrm{~nm})$ and the isoalloxazine $(\sim 515 \mathrm{~nm})$ species as reported by Song et al. ${ }^{19,20}$ We have observed that changing the emission wavelength from the peak maxima to the far red end $(580 \mathrm{~nm})$, the long component along with their relative amplitude changes (Table 2). The emission wavelength dependent lifetime decays of LCM in respective micelles are shown in Fig. 7 and S5. $\dagger$ The emission wavelength dependent lifetime decays suggests a switch over of the structural change of LCM in the micellar media.

Berr et $a{ }^{45}$ by neutron scattering method proposed that the water molecule can penetrate into the micelle upto a certain region. The compactness of the micelle headgroup increases, with increase of hydrophobic alkyl chain length of the surfactant. Thereby, degree of water penetration will be less. In other words, micellar hydration will be small. In case of anionic micelles (such as SDS) due to the smaller headgroups, probability of water penetration will be less ${ }^{46,47}$ compared to CTAB. In other words, water molecule can enter inside the micellar core depending on the compactness of the micellar units. ${ }^{46}$ In case of cationic micelles, the strong binding interaction between probe-micelles can be attributed from the fact that the $\mathrm{N}$-atom in LCM possesses negative charge, as found from quantum chemical calculation. ${ }^{36}$ This is responsible for strong binding interaction. In the Stern-layer, cationic $-\mathrm{N}\left(\mathrm{CH}_{3}\right)_{3}{ }^{+}$head groups and $\mathrm{Br}^{-}$counterions are poorly solvated compared to anionic micelles like SOS and SDS. This is due to the intervening methyl group of the positively charged head groups, which causes the inhibition of water penetration in the corresponding micelles. This statement is supported from the literature. ${ }^{48-50}$ Since the hydrophilic headgroups of cationic surfactants are same in case of DTAB, MTAB and CTAB. The only difference is their hydrophobic alkyl chain length. Due to this reason hydration of micelles retarded and becomes drier. So the binding ability increases from $\mathrm{DTAB} \rightarrow \mathrm{MTAB} \rightarrow \mathrm{CTAB}$. To ascertain the location of the probe molecule, we have measured the emission peak position of LCM in non polar isooctane solvent and found that the peak appeared at $420 \mathrm{~nm}$ (Fig. S6b $\dagger$ ). LCM exhibits very poor solubility (it may form aggregates in isooctane) and it shows low absorbance value in nonpolar isooctane solvent (Fig. S6a†). The emission peak position of LCM in isooctane is not identical with LCM in different micellar environment. We have noticed that in micellar media the fluorescence intensity at 
$420 \mathrm{~nm}$ is very low compared to their emission peak intensity. So it maybe assumed that the fluorophore resides at the micellar interfacial region (Stern-layer) and the probability of probe molecule in the micellar core region is very less (Scheme 2).

Micelles comprise of cationic surfactants (DTAB, MTAB, CTAB), the counter anion is bromide. The blue shifted emission spectra of LCM compared to that in pure water, as well as the life time value drastically decreases in the presence of cationic micelles. It is known that bromide ion may act as potent dynamical quencher and one may expect that the quenching of fluorescence of LCM is due to the presence of bromide anion. To know whether, the quenching of fluorescence of LCM is due

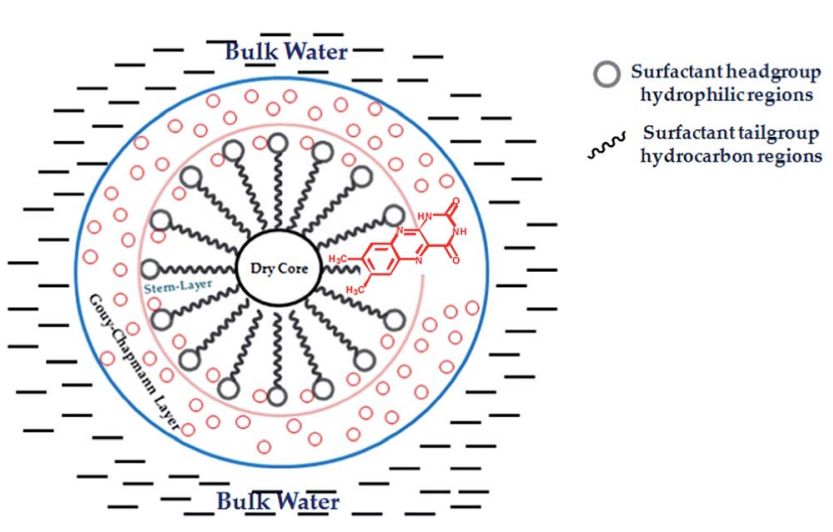

Scheme 2 Schematic representation of LCM in micellar media. to the cationic micellar environment or due to the presence of bromide anion. We have studied the photophysics LCM in presence of benzyl dimethyl hexadecyl ammonium chloride (BHDC) surfactant and also in presence of several salts such as $\mathrm{KBr}$, tetramethyl ammonium bromide (TMAB), $\mathrm{KCl}$ and tetramethyl ammonium chloride (TMAC). We have observed that with successive addition of BHDC beyond the CMC value, the fluorescence intensity, quantum yield and the fluorescence lifetime values gradually decreases. In presence of BHDC surfactants (concentration $\sim 20$ times CMC), the average fluorescence lifetime value becomes $1.10 \mathrm{~ns}$. In addition, we have found similar type of lifetime components, as observed in the case of other cationic surfactants. The fluorescence intensity as well as the lifetime value significantly affected in presence of higher concentration $[1(\mathrm{M})]$ of bromide and chloride salts (Fig. S7 and S8†). Previously, Biczók and co-workers reported ${ }^{23}$ that the photophysics of the LCM molecule changes in presence of the fluoride $\left(\mathrm{F}^{-}\right)$and acetate anion $\left(\mathrm{CH}_{3} \mathrm{COO}^{-}\right)$. The fluorescence intensity significantly decreases in presence of the anion. ${ }^{23}$ We have also found similar results in presence of bromide and chloride anions. We have found that in presence of $1(\mathrm{M})(\mathrm{KBr}, \mathrm{TMAB}, \mathrm{KCl}$ and TMAC) salts, both the fluorescence intensity and lifetime values are significantly quenches (Table $\mathrm{S} 1 \dagger)$. We have also noticed that in presence of $\mathrm{KBr}$ and TMAB salts (where $\mathrm{Br}^{-}$is counter anion) the quenching efficiency is comparatively high as compared to the $\mathrm{KCl}$ and TMAC salts (where $\mathrm{Cl}^{-}$is counter anion). This increase of quenching efficiency in the presence of bromide ion is due to the effect, that
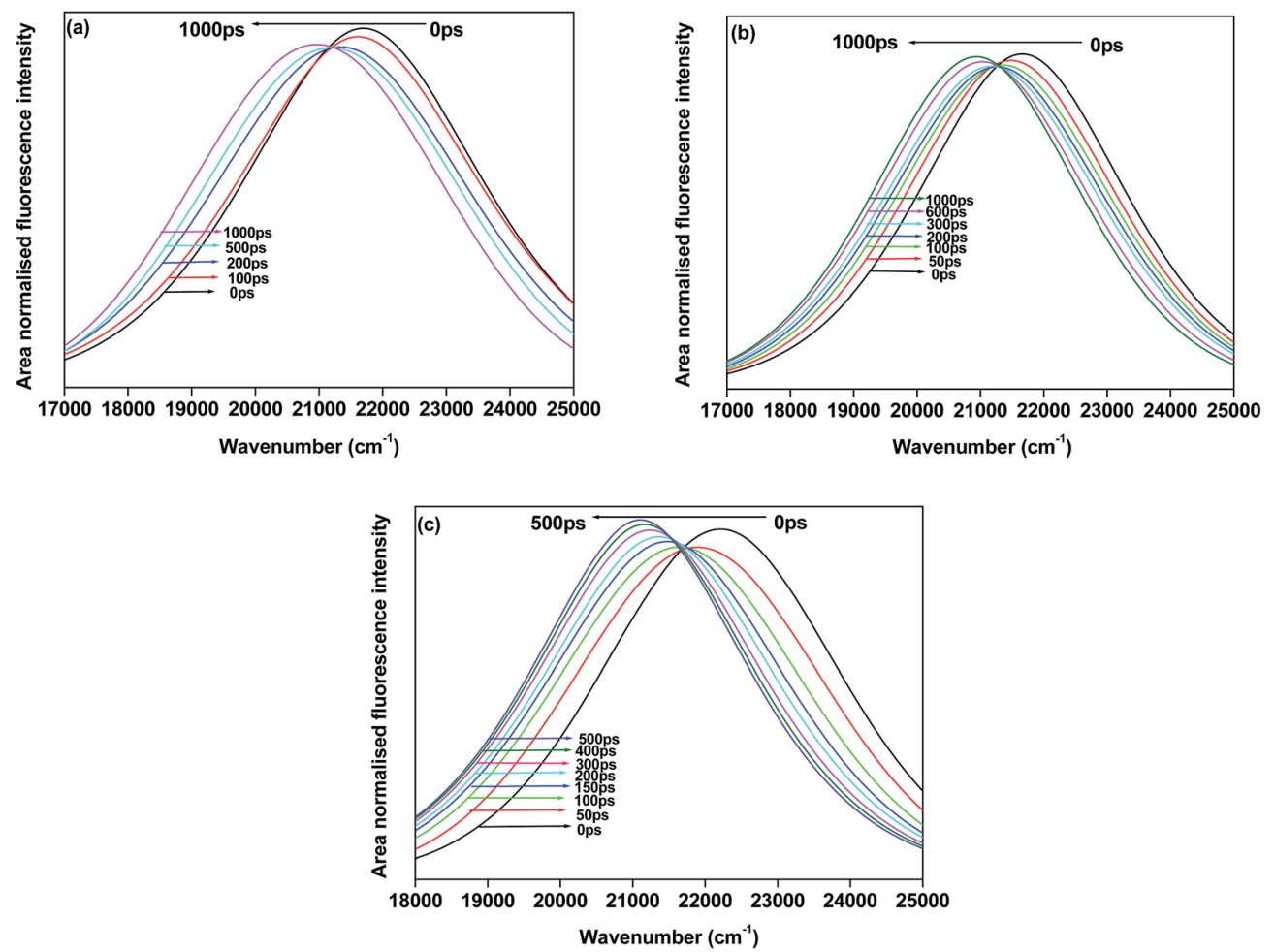

Fig. 8 The time resolved area normalised emission spectra (TRANES) of LCM in (a) MTAB and (b) CTAB and (c) DTAB micellar environments ( $\lambda_{\text {exi }}=$ $375 \mathrm{~nm})$ 
bromide ion may also acts as a potent quenching source. In presence of micelles we have found three lifetime components (Table 2 and S1 $\dagger$ ). Therefore, from this observation we can demonstrate that the emission behaviour of LCM molecule in the presence of micelles are different compared to that in salts.

\subsection{Time resolved area normalised emission spectra (TRANES)}

To explicate different emissive species present in the micellar systems and to perceive the interrelation between them, we have constructed the time resolved area normalised emission spectra (TRANES) using the procedure as reported by Periasamy and coworkers. ${ }^{51,52}$ They proposed that the presence of an isoemissive point in TRANES depicts the two distinct emissive species in the excited state. ${ }^{51,52}$ In present study, we have found a clear isoemissive point present in cationic micellar media. The TRANES shows isoemissive point at $21190 \mathrm{~cm}^{-1}, 21270 \mathrm{~cm}^{-1}, 21700$ $\mathrm{cm}^{-1}$ in the case of DTAB, MTAB and CTAB micelles respectively. The TRANES of LCM in the presence of cationic micellar environments are shown in Fig. 8. So, from TRANES analysis, we have inferred that the emission arises from two distinct species of LCM. We have also found the isoemissive point of LCM in our previous results. ${ }^{34}$

\section{Conclusion}

In this study, the photophysical properties of LCM in anionic and cationic micellar environments have been reported, using the UV-Vis absorption, steady-state and time-resolved fluorescence spectroscopy. The ground state and excited state maxima position of LCM are modified in the presence of micelles. The experimental results suggest the structural dynamism of LCM in micelles. The spectral behaviour of LCM is dependent on both the excitation and emission wavelength. Variation of alkyl chain length of the hydrophobic tail group of surfactants along with compactness of head group, play an important role and changes the ground state and excited state characteristics as well as binding constant values and free energy changes. The excitation wavelength dependent fluorescence emission behaviour of LCM was observed in micellar media. It is due to the presence of two different species of LCM. The time resolved area normalised emission spectra (TRANES) reveals the presence of an isoemissive point. This clearly depicts existence the two distinct emissive species.

\section{Acknowledgements}

All authors are thankful to IIT Patna, India for research facilities. B. M is thankful to IIT Patna for research fellowship. A. C. is thankful to IIT Patna and CSIR, New Delhi for research fellowship.

\section{References}

1 M. G. Khaledi and E. D. Breyer, Anal. Chem., 1989, 61, 1040. 2 K. Kalyanasundaram, Chem. Soc. Rev., 1978, 7, 453.
3 Photochemistry in organised and constrained media, ed. V. Rammurthy, VCH, New York, 1991.

4 H. Alkan-Oxynksel, S. Ramkrishnan, H. B. Chai and J. M. Pezzuto, Pharm. Res., 1994, 11, 206.

5 Z. Yuan and F. Liang, Curr. Org. Chem., 2014, 18, 2016.

6 A. Mallick, P. Das and N. Chattopadhyay, J. Photochem. Photobiol., C, 2010, 11, 47.

7 R. Sabate, M. Gallardo and J. Estelrich, J. Colloid Interface Sci., 2001, 233, 205.

8 M. H. Gehlen and F. C. De Schryver, Chem. Rev., 1993, 93, 199.

9 N. J. Turro, M. Gratzel and A. M. Braud, Angew. Chem., Int. Ed., 1980, 19, 675.

10 Y. Tamoto, H. Segawa and H. Shirota, Langmuir, 2005, 21, 3757.

11 G. N. Dhembre, R. S. Moon and R. V. Kshirsagar, Int. J. Pharmacol. Biol. Sci., 2011, 2, 109.

12 M.-C. Jones and J.-C. Leroux, Eur. J. Pharm. Biopharm., 1999, 48, 101.

13 F. Muller, Topics in Current Chemistry, Springer-Verlag, Berlin, 1983, vol. 108, pp. 71-107.

14 V. Nandwana, I. Samuel, G. Cooke and V. M. Rotello, Acc. Chem. Res., 2013, 46, 1000.

15 A. Onu, M. Palamaru, E. Tutovan and C. Ciobanu, Polym. Degrad. Stab., 1998, 60, 465.

16 O. Cunningham, M. G. Gore and T. J. Mantle, Biochem. J., 2000, 345, 393.

17 J. Koziol, Photochem. Photobiol., 1996, 5, 41.

18 A. Tyagi and A. Penzkofer, Photochem. Photobiol., 2011, 87, 524.

19 P. S. Song, M. Sun, A. Koziolowa and J. Koziol, J. Am. Chem. Soc., 1974, 96, 4319.

20 J. D. Choi, R. D. Fugate and P. S. Song, J. Am. Chem. Soc., 1980, 102, 5293.

21 E. Sikorska, A. Koziołowa, M. Sikorski and A. Siemiarczuk, J. Photochem. Photobiol., A, 2003, 157, 5.

22 Z. Miskolczy, L. Biczók and H. Gorner, J. Photochem. Photobiol., A, 2009, 207, 47.

23 Z. Miskolczy and L. Biczók, Chem. Phys. Lett., 2005, 411, 238. 24 E. Sikorska, I. Khmelinskii, M. Kubicki, W. Prukała, M. Hoffmann, I. F. Machado, L. F. V. Ferreira, J. Karolczak, D. R. Worrall, A. Krawczyk, M. Insińska-Rak and M. Sikorski, J. Phys. Chem. A, 2006, 110, 4638.

25 N. Laser and J. Feitelson, Photochem. Photobiol., 1977, 25, 451. 26 D. Prukała, E. Sikorska, J. Koput, I. Khmelinskii, J. Karolczak, M. Gierszewski and M. Sikorski, J. Phys. Chem. A, 2012, 116, 7474.

27 M. Sikorski, E. Sikorska, A. Koziolowa, R. G. Moreno, J. L. Bourdelandec, R. P. Steer and F. Wilkinson, J. Photochem. Photobiol., B, 2001, 60, 114.

28 N. S. Moyon and S. Mitra, J. Phys. Chem. A, 2011, 115, 2456. 29 E. Sikorska, I. Khmelinskii, W. Prukała, S. L. Williams, M. Patel, D. R. Worrall, J. L. Bourdelande, J. Koput and M. Sikorski, J. Phys. Chem. A, 2004, 108, 1501.

30 E. Sikorska, I. V. Khmelinskii, M. Kubicki, W. Prukała, G. Nowacka, A. Siemiarczuk, J. Koput, L. P. V. Ferreira and M. Sikorski, J. Phys. Chem. A, 2005, 109, 1785. 
31 M. Marchena, M. Gil, C. Martin, J. A. Organero, F. Sanchez and A. Douhal, J. Phys. Chem. B, 2011, 115, 2424.

32 M. Gil, Y. Wang and A. Douhal, J. Photochem. Photobiol., A, 2012, 234, 146.

33 B. Tyrakowska, P. I. H. Bastiaens, A. Koziolowa and A. J. W. G. Visser, J. Photochem. Photobiol., A, 1993, 72, 235.

34 B. Maity, A. Chatterjee and D. Seth, Chem. Phys. Lett., 2013, 565, 108.

35 G. A. Crosby and J. N. Demas, J. Phys. Chem., 1971, 75, 991.

36 J. Komasa, J. Rychlewski and J. Koziol, J. Mol. Struct.: THEOCHEM, 1988, 170, 205.

37 A. Mohr, T. P. Vila, H.-G. Korth, H. Rehage and R. Sustmann, ChemPhysChem, 2008, 9, 2397.

38 S. Yamaguchi, K. Bhattacharyya and T. Tahera, J. Phys. Chem. C, 2011, 115, 4168.

39 R. F. Chen, Anal. Biochem., 1967, 19, 374.

40 J. A. Zoltewicz and S. Munoz, J. Phys. Chem., 1986, 90, 5820.

41 K. Kalyanasundaram, Photochemistry in Microheterogeneous Systems, Academic press, New York, 1987.
42 R. Ranganathan, L. Tran and B. L. Bales, J. Phys. Chem. B, 2000, 104, 2260.

43 K. Weidemaier, H. L. Tavernier and M. D. Fayer, J. Phys. Chem. B, 1997, 101, 9352.

44 G. Krishnamoorthy and S. K. Dogra, J. Colloid Interface Sci., 1999, 213, 53.

45 S. Berr, R. R. M. Jones and J. S. Johnson Jr, J. Phys. Chem., 1992, 96, 5611.

46 B. K. Paul, D. Ray and N. Guchhait, J. Phys. Chem. B, 2012, 116, 9704.

47 K. Kalyanasundaram and J. K. Thomas, J. Am. Chem. Soc., 1977, 99, 2039.

48 R. Das, G. Duportail, L. Richert, A. Klymchenko and Y. Mély, Langmuir, 2012, 28, 7147.

49 N. Lebedeva and B. L. Bales, J. Phys. Chem. B, 2006, 110, 9791. 50 B. L. Bales and R. Zana, J. Phys. Chem. B, 2002, 106, 1926.

51 A. S. R. Koti and N. Periasamy, J. Chem. Phys., 2001, 115, 7094.

52 A. S. R. Koti, M. M. G. Krishna and N. Periasamy, J. Phys. Chem. A, 2001, 105, 1767. 